\title{
Statistics of low-frequency normal-mode amplitudes in an ocean with random sound-speed perturbations: Shallow-water environments
}

\author{
John A. Colosi \\ Department of Oceanography, Naval Postgraduate School Monterey, California 93943 \\ Timothy F. Duda and Andrey K. Morozov \\ Department of Applied Ocean Physics and Engineering, Woods Hole Oceanographic Institution, Woods Hole, \\ Massachusetts 02543
}

(Received 21 December 2010; revised 6 July 2011; accepted 7 July 2011)

\begin{abstract}
Second- and fourth-moment mode-amplitude statistics for low-frequency ocean sound propagation through random sound-speed perturbations in a shallow-water environment are investigated using Monte Carlo simulations and a transport theory for the cross-mode coherence matrix. The acoustic observables of mean and mean square intensity are presented and the importance of adiabatic effects and cross-mode coherence decay are emphasized. Using frequencies of 200 and $400 \mathrm{~Hz}$, transport theory is compared with Monte Carlo simulations in a canonical shallow-water environment representative of the summer Mid-Atlantic Bight. Except for ranges less than a horizontal coherence length of the sound structure, the intensity moments from the two calculations are in good agreement. Corrections for the short range behavior are presented. For these frequencies the computed mode coupling rates are extremely small, and the propagation is strongly adiabatic with a rapid decay of cross-mode coherence. Coupling effects are predicted to be important at kilohertz frequencies. Decay of cross-mode coherence has important implications for acoustic interactions with nonlinear internal waves: For the case in which the acoustic path is not at glancing incidence with a nonlinear internal-wave front, adiabatic phase randomizing effects lead to a significantly reduced influence of the nonlinear waves on both mean and mean square intensity. [DOI: 10.1121/1.3666002]
\end{abstract}

PACS number(s): 43.30.Bp, 43.30.Re, 43.60.Cg [DRD]

Pages: 1749-1761

\section{INTRODUCTION}

Acoustical fields in shallow ocean areas invariably fluctuate because of the dynamic environment. Both the mean fields and the fluctuations themselves are of intrinsic interest. Fluctuations are caused by the time-evolving spatially complex water column and by time-dependent sound interaction with the rough and inhomogeneous boundaries. For sound at hundreds of hertz, progress on this problem has been facilitated by full-physics simulation (Monte Carlo) techniques (Headrick et al., 2000; Tielburger et al., 1997; and Fredricks et al., 2005, to mention a few). However, comparison between such simulations and results from reduced-physics analytical theory are sparse (Creamer, 1996; Chen et al., 2005), so that insight gained from such reduced-physics theories is not readily available. This paper seeks to fill this void (at least partially) by utilizing a new transport theory governing the range evolution of the mode amplitude coherence matrix (Colosi and Morozov, 2009) in a study using Monte Carlo simulations with waves having a spatially homogenous spectrum, and additional isolated deterministic waves, such as shallow water internal solitary waves (ISWs). It is important to point out that previous transport theories (Creamer, 1996) from which the present theory is based could only solve for the mode energies (i.e., the diagonal of the mode amplitude coherence matrix) under the assumption of zero cross-mode coherence. Other theoretical studies have examined cross-mode coherence (Beran and Frankenthal,
1992; Sazontov et al., 2002; Voronovich and Ostachev, 2009), but Monte Carlo simulations were not used to test the approximations. Thus understanding and quantifying the mechanisms at work in the Monte Carlo simulation via the transport theory forms the basis of our new results, and the associated validation of the transport theory builds confidence in using the theory as a tool to explain experimental results suffering from incompletely constrained environmental parameters.

In this paper a simple canonical environment typical of the summer Mid-Atlantic Bight (see Fig. 1) is treated, which is based on observations from the Shallow Water 2006 (SW06) experiment (Lynch and Tang, 2008; Colosi et al., 2012). Here the diffuse random sound-speed perturbations are modeled using a modified Garrett-Munk (GM) (Munk, 1981) internal-wave spectrum, ${ }^{1}$ and the nonlinear waves are modeled using the common hyperbolic secant form. The choice of a canonical and geographically specific environment to test an analytic model creates the possibility that the results are not robust or in some sense not universal. Despite this, a single canonical environment was chosen so that the present results can be easily reproducible by others. The issue of robustness can be resolved with further work. The present results are intended to be a benchmark as much as a validation study.

With this proper context in mind, the basic results for propagation through the random waves in our canonical environment are as follows. (1) For 200 and $400 \mathrm{~Hz}$ sound, 

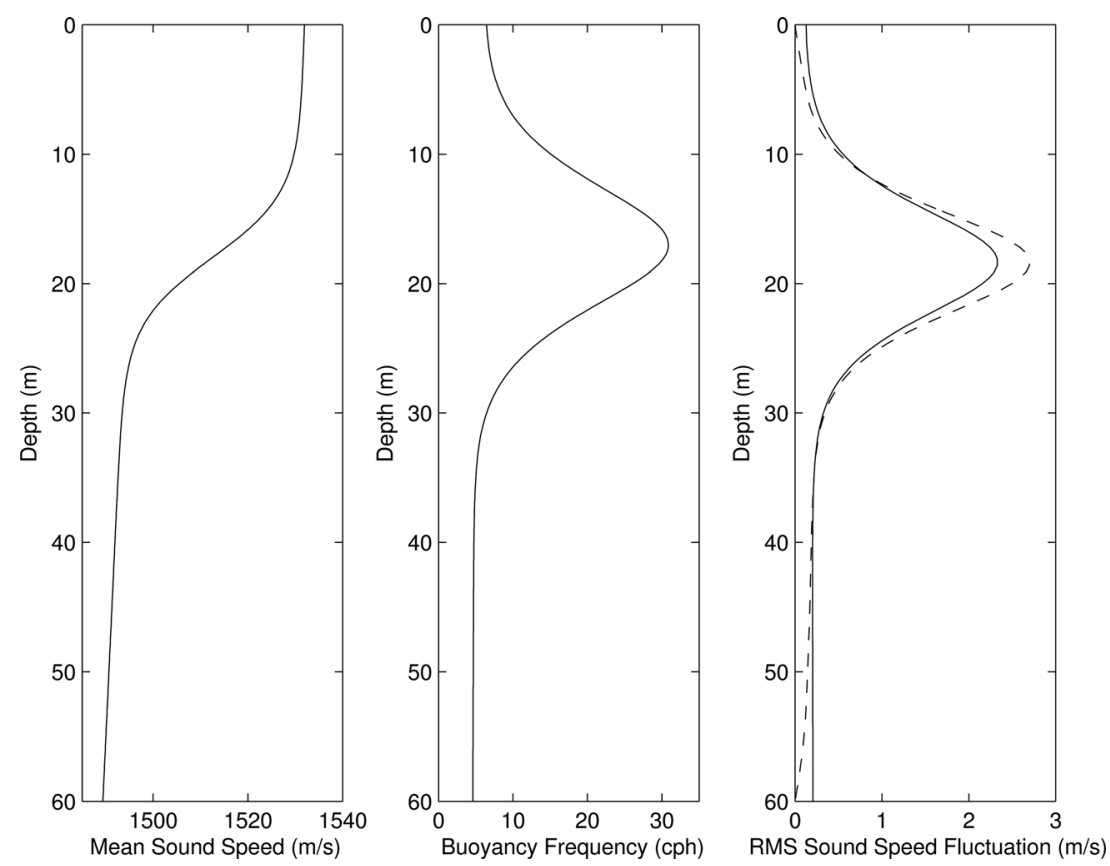

FIG. 1. Profiles of mean sound speed, buoyancy frequency, and rms sound-speed fluctuation used in the numerical calculations of this paper. For rms sound speed, the input profile is shown using a solid line, and the actual modeled profile averaging over 256 realizations is shown with a dash.

the transport theory works well for predicting the mode coherence (auto- and cross-correlation) matrix. Because of this predictive ability, theoretical estimates of mean intensity are within a fraction of a decibel of Monte Carlo results. (2) The theory allows for a quantitative estimation of the mode coupling rates and the degree of adiabaticity of the propagation. (3) For the tens of kilometer ranges of interest in shallow water, one can understand much of the propagation physics through the random waves with the simple adiabatic approximation. In fact, adiabatic estimates of second- and fourthmode amplitude moments lead to predictions of scintillation index that are in excellent agreement with Monte Carlo results. (4) The theory/Monte Carlo comparisons are sufficiently precise that an important correction to the adiabatic theory is found to be necessary. Near the source and receiver the standard theory overcounts the scattering in a so-called edge effect (Van Kampen, 1981). A new adiabatic calculation that properly treats the edges produces cross-mode coherence estimates that are in even better agreement with the Monte Carlo simulations. (5) When strong packets of ISWs are added to the Monte Carlo simulation it is found that mean intensity and intensity variance are not changed significantly by these waves. This surprising effect is the result of modal phase randomization by the diffuse waves and the corresponding loss of coherent scattering effects by the ISWs. The conclusions related to nonlinear internal waves apply when the acoustic path is not at glancing incidence to the nonlinear wave front.

There have been a few studies of this acoustical situation. A Monte Carlo simulation study of the effects of these two wave types was carried out by Tielburger et al. (1997). The present study complements that seminal work because of the extra insight provided by the transport theory and the mode cross correlations. A related study was also performed by Chen et al. (2005), who treated waves with a homogeneous spectrum in a two-layer ocean and tested a scattering theory based on the Rayleigh-Born approximation (Ratilal and Makris, 2005). Our results corroborate and serve to explain the mechanisms at work in those studies.

The outline of this paper is as follows. Section II briefly summarizes the coupled mode theory from Colosi and Morozov (2009) and presents new results for the scattering matrices, which utilize the GM spectrum. Section III shows comparisons of the theory to Monte Carlo numerical simulations of sound propagation through random fields on linear internal waves. The topic of the combined effects of random linear internal waves and nonlinear internal solitary wave packets is taken up in Sec. IV. Summary and conclusions are presented in Sec. V.

\section{TRANSPORT THEORY: MODE AMPLITUDE SECOND MOMENTS}

In this work the acoustic pressure at frequency $\omega$ is expressed as a $2 \mathrm{D}$ normal mode expansion with the unperturbed modes (Creamer, 1996; Colosi and Morovoz, 2009),

$$
p(r, z ; \omega)=\sum_{n=1}^{N} \frac{a_{n}(r) \phi_{n}(z)}{\sqrt{k_{n} r}} .
$$

In this representation, all the variability is contained in the complex mode amplitude $a_{n}(r)$. The eigenmodes $\phi_{n}(z)$ and eigenwavenumbers $k_{n}$ are from the unperturbed mode equation, $\rho_{0}(z) \partial / \partial z\left(\rho_{0}^{-1}(z) \partial \phi_{n} / \partial z\right)+\left(\vec{k}^{2}(z)-k_{n}^{2}\right) \phi_{n}=0$, where the background density is $\rho_{0}(z)$, and the $2 \mathrm{D}$ sound-speed field is represented by $c(r, z)=\bar{c}(z)+\delta c(r, z)$, with $\bar{k}(z)=\omega / \bar{c}(z)$. The evolution equations for the mode amplitudes $a_{n}(r)$ are given by the well-known set of coupled ordinary differential equations (Dozier and Tappert, 1978a; Dozier, 1983) 


$$
\frac{d a_{n}}{d r}-i l_{n} a_{n}=-i \sum_{m=1}^{N} \rho_{m n}(r) a_{m}(r),
$$

where the symmetric coupling matrix $\rho_{m n}(r)$ is given by

$$
\rho_{m n}(r)=\frac{k_{0}^{2}}{\sqrt{k_{n} k_{m}}} \int_{0}^{D} \frac{\phi_{n}(z) \phi_{m}(z)}{\rho_{0}(z)} \mu(r, z) d z .
$$

Here $\mu(r, z)=\delta c(r, z) / c_{0}$ is the fractional sound-speed perturbation, $k_{0}=\omega / c_{0}$ is a representative wavenumber, $D$ is the water depth, and the complex modal wave number is $l_{n}=k_{n}+i \alpha_{n}$. Typically the complex part of the wavenumber $\alpha_{n}$ is obtained by perturbation methods (Jensen et al., 1993).

An important acoustic observable is the mean intensity given by

$$
\begin{aligned}
\langle I(r, z)\rangle & =\left\langle|p(r, z)|^{2}\right\rangle \\
& =\sum_{n=1}^{N} \sum_{p=1}^{N} \frac{\left\langle a_{n} a_{p}^{*}(r)\right\rangle}{r} \frac{\phi_{n}(z) \phi_{p}(z)}{\sqrt{k_{n} k_{p}}}
\end{aligned}
$$

and the relation to the cross-mode coherence, $\left\langle a_{n} a_{p}^{*}(r)\right\rangle$, is seen. In the limit of uncorrelated modes, the mean intensity is an incoherent sum given by

$$
\langle I(r, z)\rangle=\sum_{n=1}^{N} \frac{\left\langle\left|a_{n}\right|^{2}(r)\right\rangle}{r} \frac{\phi_{n}^{2}(z)}{k_{n}},
$$

where it is evident that the modal interference pattern is lost. It will be demonstrated that the incoherent modes case will be important for long-range propagation in shallow water, and it will be important when interaction with nonlinear internal waves are considered.

Using the small-angle forward scattering and Markov approximations, Creamer (1996) and Colosi and Morozov (2009) show that a cross-mode coherence transport equation for propagation though random fields of linear internal-wave induced sound-speed perturbations is

$$
\begin{aligned}
\frac{d\left\langle a_{n} a_{p}^{*}\right\rangle(r)}{d r}= & i\left(l_{n}-l_{p}^{*}\right)\left\langle a_{n} a_{p}^{*}\right\rangle-\sum_{m=1}^{N} \sum_{q=1}^{N}\left(\left\langle a_{q} a_{p}^{*}\right\rangle I_{m n, q m}\right. \\
& -\left\langle a_{m} a_{q}^{*}\right\rangle I_{m n, q p}^{*}-\left\langle a_{q} a_{m}^{*}\right\rangle I_{m p, q n} \\
& \left.+\left\langle a_{n} a_{q}^{*}\right\rangle I_{m p, q m}^{*}\right)
\end{aligned}
$$

with the scattering matrix $I_{m n, q p}$ given by

$$
I_{m n, q p}=\int_{0}^{\infty} d \xi \Delta_{m n, q p}(\xi) e^{i l_{p q} \xi} .
$$

Here $\Delta_{m n, q p}(\xi)=\left\langle\rho_{m n}(r) \rho_{q p}(r+\xi)\right\rangle$ is the horizontal correlation function of the coupling matrices between modes $m, n$ and $q, p$, and $l_{p q}=l_{p}-l_{q}$. The interested reader can refer to Creamer (1996) and Colosi and Morozov (2009) for details of the derivation. An important limitation of Eq. (6) is that the results will only be accurate when the final range is greater than a few horizontal correlation lengths of the internal waves. Although this issue is not so critical in deepwater long-range acoustic propagation it will be much more important in this shallow-water problem (see discussion and results from Sec. III C). Note that a somewhat more complicated 3D result for deep water has been obtained by Voronovich and Ostashev (2009).

Previous work in deep water used an idealized form of the internal-wave spectrum such that an analytic form of the correlation function $\Delta_{m n, q p}$ and, thus, the scattering matrix could be obtained (Colosi and Morozov, 2009). Here a new form of the coupling matrix horizontal correlation function is used, which assumes an arbitrary horizontally isotropic spectrum $S_{j}(k)$ and it is found that

$$
\Delta_{m n, q p}(\xi)=\sum_{j=1}^{J_{m}} G_{m n}(j) G_{q p}(j) \int_{0}^{\infty} d k S_{j}(k) J_{0}(k|\xi|),
$$

where $J_{0}$ is the zeroth-order Bessel function, $k$ is the magnitude of the horizontal wave number, $j$ is the internal-wave mode number, $J_{m}$ is the maximum internal-wave mode number, and

$$
\begin{aligned}
G_{m n}(j)= & k_{0}^{2} \sqrt{\frac{2}{k_{n} k_{m}}} \int_{0}^{D} d z\left\langle\mu^{2}(z)\right\rangle^{1 / 2} \\
& \times \sin [\pi j \hat{z}(z)] \frac{\phi_{n}(z) \phi_{m}(z)}{\rho_{0}(z)} .
\end{aligned}
$$

In Eq. (9), $\hat{z}(z)$ is the Wentzel-Kramers-Brillouin (WKB) stretched vertical coordinate (Colosi and Brown, 1998), and

$$
\left\langle\mu^{2}(z)\right\rangle=\frac{\zeta_{0}^{2}}{c_{0}^{2}} \frac{N_{0}}{N(z)}\left(\frac{d c}{d z}\right)_{p}^{2}
$$

is the fractional sound-speed variance. In Eq. (10), the buoyancy frequency profile is $N(z)$, with $N_{0}=3$ cycles per hour (cph) being a reference buoyancy frequency, the reference displacement is $\zeta_{0}$, and the potential sound-speed gradient is $(d c / d z)_{p}$. Plugging Eq. (8) into Eq. (7) and neglecting the modal attenuation factor $\alpha_{p q}$ the following new instructive result is obtained:

$$
I_{m n, q p}=\sum_{j=1}^{J_{m}} G_{m n}(j) G_{q p}(j) \int_{0}^{\infty} d k \frac{S_{j}(k)}{\sqrt{k^{2}-k_{q p}^{2}}} .
$$

The neglect of $\alpha_{p q}$ in Eq. (7) is justified if the correlation length of $\Delta_{m n, q p}$ is less than $1 / / \alpha_{p q} /$ (Creamer, 1996). Through numerical calculations and analytical considerations (see the following) it has been found that this approximation is quite good. In this paper the wavenumber integral in Eq. (11) is done numerically.

Equation (11) provides important information concerning the acoustically relevant ocean scales for acoustic scattering, namely the integrable singularity at $k=k_{q p}$ means that the scattering will be strongly influenced by the magnitude of the spectrum in the neighborhood of the difference wavenumber. The adiabatic terms in the scattering matrix 
(i.e., $\left.I_{n n, p p}\right)$ will be important in this analysis. Here the difference wavenumber is zero and the contribution to the scattering matrix from the various internal-wave horizontal wavenumbers is $S_{j}(k) / k$ which typically strongly emphasizes the large scale ocean structure. The importance of the ocean structure at the difference wavenumber has been emphasized by many previous authors (Dozier and Tappert, 1978a; Creamer 1996; Zhou et al., 1991; Rouseff and Tang, 2006; Colosi, 2008).

In this paper the GM internal-wave spectrum is utilized,

$$
S_{j}(k)=H(j) \frac{4}{\pi} \frac{k^{2} k_{j}}{\left(k^{2}+k_{j}^{2}\right)^{2}},
$$

where $k_{j}=\pi f j / N_{0} B, f$ is the Coriolis parameter, and $N_{0} B=\int_{0}^{D} N(z) d z$. Here $H(j)=N_{j} /\left(j^{2}+j_{*}^{2}\right)$ is the GM vertical mode number spectrum with $N_{j}$ being the normalization. Note here that the spectrum is normalized to unity, and the WKB depth scaling of the internal-wave displacements has been subsumed into Eq. (10). To first order for the GM spectrum, the horizontal correlation length of mode $j$ is $1 / k_{j}$, and, thus, the condition for the validity of Eq. (11) is $\left|\alpha_{p q}\right| / k j \ll 1$. Importantly, measurements from the SW06 experiment (Colosi et al., 2012) show that the random internal waves have a frequency and mode spectrum that is quite close to the GM model except the modal band width parameter $j_{*} \simeq 1$.

It should be pointed out that the WKB approximation for the internal-wave dispersion relation and normal modes have been made in this analysis for the sake of analytic and numerical simplicity. The WKB modes are seen in Eq. (9) and the WKB dispersion relation is used to translate the internal-wave frequency spectrum into a wavenumber spectrum [Eq. (12)]. Although it is true that WKB analysis is central to the deep water GM spectral model, the applicability of this approximation in shallow water has not been tested; in fact our knowledge of the shallow-water internal-wave spectrum is somewhat limited. It should also be remarked that the WKB analysis is not essential to the transport equation theory, and thus the scattering matrices could be easily modified to use true linear internal wave modes and dispersion relation, as was done by Voronovich and Ostachev (2009) and Rouseff and Tang (2006).

\section{A. Mode energy}

Colosi and Morozov (2009) have shown that the evolution of mode energy in Eq. (6) is insensitive to the crossmode coherence terms. When these coherence terms are neglected a considerably simpler transport equation for mode energy is obtained (Creamer, 1996), which is of the form

$$
\begin{aligned}
\frac{\partial\left\langle\left|a_{n}\right|^{2}\right\rangle}{\partial r}= & -2 \alpha_{n}\left\langle\left|a_{n}\right|^{2}\right\rangle \\
& +\sum_{m=1}^{N} 2 \operatorname{Re}\left(I_{m n, m n}\right)\left(\left\langle\left|a_{m}\right|^{2}\right\rangle-\left\langle\left|a_{n}\right|^{2}\right\rangle\right) .
\end{aligned}
$$

Considerable use of this result will be made in the following analysis as it provides a simple means to predict small mode coupling effects seen in the Monte Carlo simulations.

\section{B. Adiabatic approximation}

Another important aspect of the analysis here will be the adiabatic approximation in which the scattering matrices are assumed zero except for those of the form $I_{n n, p p}$. The adiabatic solutions to Eq. (6) are thus,

$$
\begin{aligned}
& \left\langle\left|a_{n}\right|^{2}\right\rangle(r)=\left\langle\left|a_{n}\right|^{2}\right\rangle(0) e^{-2 \alpha_{n} r}, \\
& \left\langle a_{n} a_{p}^{*}\right\rangle(r)=\left\langle a_{n} a_{p}^{*}\right\rangle(0) e^{i\left(l_{n}-l_{p}^{*}\right) r} e^{-\left(I_{n n, n n}-2 I_{n n, p p}+I_{p p, p p}\right) r} .
\end{aligned}
$$

It is important to note that Eq. (14), derived from the transport equation, is exactly what one would obtain if a traditional adiabatic approximation (Jensen et al., 1993) was made on the wave equation; that is to say the approximations of the transport equation preserve the traditional adiabatic result. Further, in the adiabatic limit the scattering matrices with the GM spectrum have an analytic form such that

$$
I_{n n, p p}=\frac{2}{\pi} \sum_{j=1}^{J_{m}} H(j) \frac{G_{n n}(j) G_{p p}(j)}{k_{j}} .
$$

The adiabatic decorrelation range $R_{n, p}$ for modes $n$ and $p$ can thus be defined using Eqs. (14) and (15) giving

$$
\begin{aligned}
R_{n, p} & =\left(\frac{I}{I_{n n, n n}-2 I_{n n, p p}+I_{p p, p p}}\right) \\
& =\frac{\pi}{2}\left(\sum_{j=1}^{J_{m}} \frac{H(j)}{k_{j}}\left(G_{n n}(j)-G_{p p}(j)\right)^{2}\right)^{-1} .
\end{aligned}
$$

\section{MONTE CARLO SIMULATION RESULTS}

Monte Carlo numerical simulations of mode propagation through random realizations of internal-wave induced sound-speed perturbations were carried out to test the accuracy of the cross-mode coherence transport equation [Eq. (6)], and to delve into the important acoustic propagation physics related to mean intensity. The issue of the combined effects of random internal waves and nonlinear internal-wave packets is taken up in Sec. IV.

\section{A. Description of the calculations}

In this numerical example, calculations are presented utilizing a 2D sound-speed field of the form $c(r, z)=\bar{c}(z)$ $+\delta c(r, z)$, where $\bar{c}(z)$ is the background sound-speed profile and $\delta c$ is a random linear internal-wave perturbation. The ocean acoustic environment for these calculations is inspired by the SW06 experiment (Lynch and Tang, 2008; Colosi et al., 2012). For the background profiles, the ocean is assumed to have a constant salinity of 35 psu (practical salinity unit) and a mean temperature profile of the form 


$$
\bar{T}(z)=T_{0}-0.04 z-\frac{\Delta T}{2}\left[1+\tanh \left(\frac{z-z_{\text {th }}}{\Delta_{\mathrm{th}}}\right)\right]
$$

with parameters $T_{0}=24^{\circ} \mathrm{C}, \Delta T=12{ }^{\circ} \mathrm{C}$, a thermocline depth of $z_{\mathrm{th}}=17.6 \mathrm{~m}$, and a thermocline width of $\Delta_{\mathrm{th}}=5 \mathrm{~m}$. The water depth, $D$, is taken to be $60 \mathrm{~m}$. Using the equation of state of sea water, the salinity and temperature profiles are used to compute the mean sound speed $\bar{c}(z)$ and buoyancy frequency $N(z)$ profiles which are shown in Fig. 1. The background density is fixed in the water column and seabed with values of 1000 and $1500 \mathrm{~kg} / \mathrm{m}^{3}$, respectively. In the seabed an attenuation constant of $\alpha^{\lambda}=0.2 \mathrm{~dB} / \lambda$ and a sound speed of $c_{b}=1700 \mathrm{~m} / \mathrm{s}$ are used.

Random realizations of linear internal-wave induced sound-speed perturbations, $\delta c(r, z)$, are modeled using the GM internal-wave spectrum and the method of Colosi and Brown (1998). Parameters for the internal-wave fluctuations are taken to be $\zeta_{0}=2 \mathrm{~m}, j_{*}=1$, and the latitude is $39^{\circ} \mathrm{N}$. For the present model the parameter $N_{0} B$ is equal to $1.1 \mathrm{rad} \mathrm{m} / \mathrm{s}$. These spectral values are consistent with observations from the Mid-Atlantic Bight (Yang and Yoo, 2002; Colosi et al., 2012). In our numerical calculations a maximum internalwave mode number of $J_{m}=15$ is used, and internal waves with horizontal scales from 0.05 to $300 \mathrm{~km}$ are simulated. The mode number cutoff $J_{m}$ yields a minimum internal-wave vertical wavelength of $\sim 4 \mathrm{~m}$, the scale at which one expects internal-wave shear instability to start significantly attenuating the spectrum (Munk, 1981). The input fractional soundspeed variance for the Colosi/Brown model is given by Eq. (10), where the potential sound-speed gradient is approximated by the total gradient of the mean sound-speed profile, and $c_{0}=1500 \mathrm{~m} / \mathrm{s}$. Figure 1 shows the input profile of $c_{0}\left\langle\mu^{2}(z)\right\rangle^{1 / 2}$ computed from Eq. (10), as well as the actual numerical modeled values from the Colosi/Brown method. The difference in these two curves occurs because only 15 modes are modeled and the internal-wave modes have a zero boundary condition. The magnitude of the modeled soundspeed perturbations are consistent with those observed during the SW06 experiment (Colosi et al., 2012).

Finally it should be noted that the transport theory described in Sec II and the Colosi/Brown method are completely consistent in their use of the WKB approximation and, thus, the calculations presented here provide a direct test of the acoustic propagation physics without the ambiguity of having different ocean models in the theory and Monte Carlo simulation. That being said, one may rightly question the use of the WKB approach in shallow water; Fig. 2 shows how the WKB mode functions compare to a direct calculation from the linear internal-wave mode equation. For internal-wave frequencies between $f$ and $\sim 2$ cph the WKB modes (except for mode 1 , as expected) are a good approximation to the true dynamic modes, and thus inaccuracies are only due to the higher internal-wave frequencies between 2 and $30 \mathrm{cph}$.

Last, the Monte Carlo acoustic propagation simulations were carried by numerical solution of Eq. (2) using the techniques as described in Dozier and Tappert (1978b), and Creamer (1996). For these simulations, 200 and $400 \mathrm{~Hz}$ point sources were located at a depth of $z_{s}=55 \mathrm{~m}$. The initial condition was normalized such that $a_{n}(0)=\phi_{n}\left(z_{s}\right) / N_{a}$ where $N_{a}$ is obtained from the condition $\sum_{n=1}^{N}\left\langle\left|a_{n}(0)\right|^{2}\right\rangle=1$. Here only trapped mode propagation was considered, thus, yielding maximum mode numbers of 6 and 12 for the frequencies of 200 and $400 \mathrm{~Hz}$, respectively (see Jensen et al., 1993, Eq. 2.172 for the mode cutoff frequencies for the Pekeris problem). For each frequency a total of 256 realizations were used to compute the normal mode and mean intensity statistics.

\section{B. Results: Mean intensity}

Figures 3 and 4 show comparisons of 200 and $400 \mathrm{~Hz}$ mean intensity for a receiver at $50 \mathrm{~m}$ in depth computed by Monte Carlo simulation and by using Eqs. (6) and (4). For reference the unperturbed intensity is also plotted. The agreement between theory and direct simulation is seen to be excellent past a range of $\sim 5 \mathrm{~km}$, with deviations at the level of a fraction of a decibels. The small deviations between theory and simulation are consistent with sampling errors. For ranges less than $\sim 5 \mathrm{~km}$ there are larger discrepancies particularly near the nulls, and this result is understood as an edge effect in the theory (Van Kampen, 1981); that is to say these ranges are comparable to or shorter than the horizontal correlation length of the internal waves, which is computed to be $3.2 \mathrm{~km}^{2}$ As previously stated, the theory is not expected to be accurate at these short ranges, although corrections can be applied (more later).

An important effect observed in Figs. 3 and 4 is that the random internal-wave perturbations are seen to smooth out the unperturbed modal interference pattern [see Eq. (5)] progressively with increasing range; this effect is due to the decorrelation of the modes by the random sound-speed fluctuations. The large differences between the mean intensity and the unperturbed intensity demonstrate the importance of random internal-wave induced sound-speed perturbations in affecting shallow-water acoustic fields. Due to this fact, the behavior of cross-mode coherence decay is discussed in more detail in the next section.

\section{Results: Cross-mode coherence}

Figure 5 shows the range evolution of cross-mode coherence at 200 and $400 \mathrm{~Hz}$ for a few low mode pairs from both the transport theory and the Monte Carlo simulation. To remove the effects of modal attenuation and some small measure of coupling, the coherences in Fig. 5 are normalized; i.e., the quantity $\left|\left\langle a_{n} a_{p}^{*}\right\rangle\right|(r) / \sqrt{\left\langle\left|a_{n}\right|^{2}\right\rangle(r)\left\langle\left|a_{p}\right|^{2}\right\rangle(r)}$. is presented. The coherences are seen to decay at a scale on the order of tens of kilometers, and the higher frequencies, as expected, have a more rapid decay.

Comparing the transport theory to the Monte Carlo simulation, one would expect relatively good agreement, given the comparisons obtained for mean intensity. Figure 5, however, shows the Monte Carlo simulation to be clearly more coherent than the theory, and the theory shows the decay of cross-mode coherence to be almost completely adiabatic. The discrepancy between the Monte Carlo simulation and theory, comes from the previously mentioned "edge" effect, where the transport theory improperly treats the ranges, 

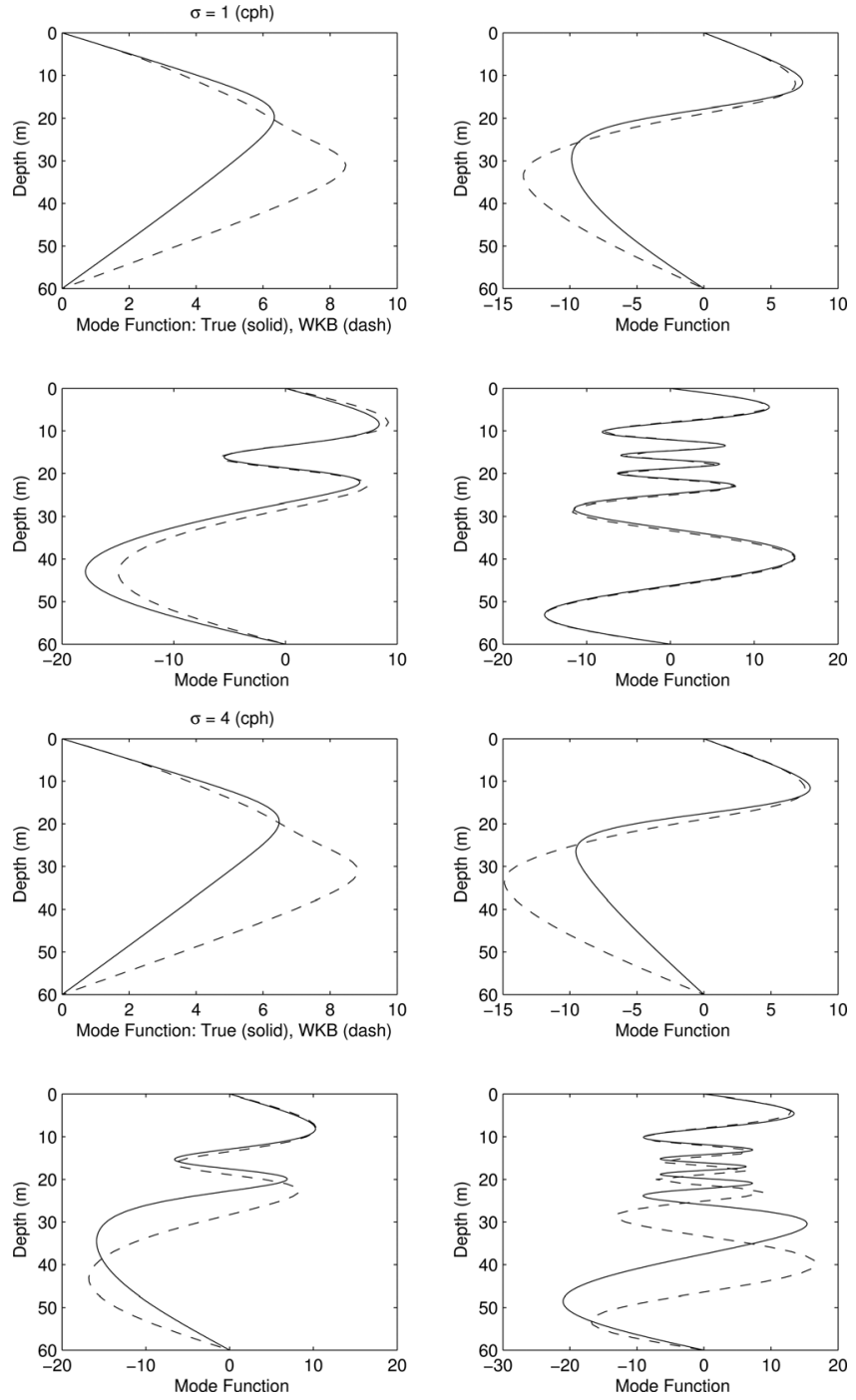

FIG. 2. Comparison of true linear internal-wave mode shapes (solid) with the WKB approximation (dash). Upper four panels are for an internal-wave frequency of $1 \mathrm{cph}$, and the lower four panels are for a frequency of $4 \mathrm{cph}$. The modes displayed are $1,2,4$, and 10 .

which are on the order of a coherence length of the internal waves. This effect can be easily understood in the adiabatic approximation, which apparently applies quite well here. From adiabatic theory, one obtains (Colosi and Morozov, 2009)

$$
\begin{aligned}
I_{n n, n n}+I_{p p, p p}-2 I_{n n, p p}= & \frac{1}{2} \int_{0}^{r} d r_{1} \int_{0}^{r} d r_{2}\left(\Delta_{n n, n n}(|\xi|)\right. \\
& \left.+\Delta_{p p, p p}(|\xi|)-2 \Delta_{n n, p p}(|\xi|)\right),
\end{aligned}
$$

where $\xi=r_{1}-r_{2}$. In the transport theory, the approximation that $r$ is much larger than the correlation length of the internal-waves allows one to write

$$
\begin{aligned}
I_{n n, n n}+I_{p p, p p}-2 I_{n n, p p} \simeq & \frac{1}{2} \int_{0}^{r} d r_{1} \int_{-\infty}^{\infty} d \xi\left(\Delta_{n n, n n}(|\xi|)\right. \\
& \left.+\Delta_{p p, p p}(|\xi|)-2 \Delta_{n n, p p}(|\xi|)\right),
\end{aligned}
$$

where it can be seen that this result exactly corresponds to that from Eq. (11). The overcounting in Eq. (19) relative to Eq. (18), where $r$ is on the order of the internal-wave correlation length is evident, and thus the transport theory predicts too rapid a decay of the coherence with range. Using Eqs. (8) and (14) with Eq. (18), cross-mode coherences with the edge's properly treated were computed and are shown in Fig. 5; the new coherences match the Monte Carlo results very well. Although results are only shown here for a few mode pairs similar results are seen for the other modes.

Thus, it is found somewhat surprisingly that the adiabatic approximation provides some very useful insight into the problem. In fact, adiabatic mean intensity curves (not shown in Figs. 3 and 4) directly overlay the full theory curves, and thus for the environment and frequencies considered here the adiabatic approach is completely adequate. Although the issue of adiabaticity is not directly addressed in previous work (Tielburger et al., 1997) the present results appear consistent with those because large sound-speed perturbations and ranges of the order of hundreds of kilometers are required for coupling to be observed. It should be noted that the adiabaticity of the propagation suggests that higher moments of the field will be quite easy to treat. This fascinating result leads to the questions of mode energies and coupling, which are discussed in the next section.

\section{Results: Mode energy}

Figure 6 shows the range evolution of mean mode energy from the Monte Carlo simulation, predictions from the mode energy transport equation [Eq. (13)], and predictions from adiabatic theory [Eq. (14)]. Here some weak effects from mode coupling are observed. At $200 \mathrm{~Hz}$ for the most part the three curves (Monte Carlo, transport, and adiabatic) are linear and directly upon one another. Here the slope of the curves is dictated by the modal attenuation factor. However, for the two weakest initial energy modes, the curves are seen to be initially linear and then level off with the Monte Carlo simulation and theory following one another. This weak effect from internal-wave coupling was previously misinterpreted as a numerical artifact (Tielburger, et al., 1997). At $400 \mathrm{~Hz}$, the coupling effects are even more evident, but still basically weak. Not only is the leveling-off effect seen, but here the two weakest excited modes show a completely different slope than the adiabatic theory would predict. The point here is not to dwell on a small effect that has virtually no impact on real observables like mean intensity, but it is to show that the theory can be used to predict coupling effects in shallow water. It must also be pointed out that coupling effects in other shallow water environments may be significantly higher than those modeled here.

The previous results have clearly shown that for the cases considered here, mode coupling rates are quite small, and that the mode energy transport equation is useful for predicting coupling effects. Writing this equation [Eq. (13)] in matrix form $d A / d r=F A$, it is clearly seen that the solutions are a set of exponentials whose evolution in range is dictated by the eigenvalues of the matrix $F$. In lossy shallow water environments where the coupling rates are small compared to the attenuation rates, the eigenvalues are strongly affected by the attenuation. So to predict coupling rates the attenuation constants $\alpha_{n}$ in Eq. (13) are set to zero and the 

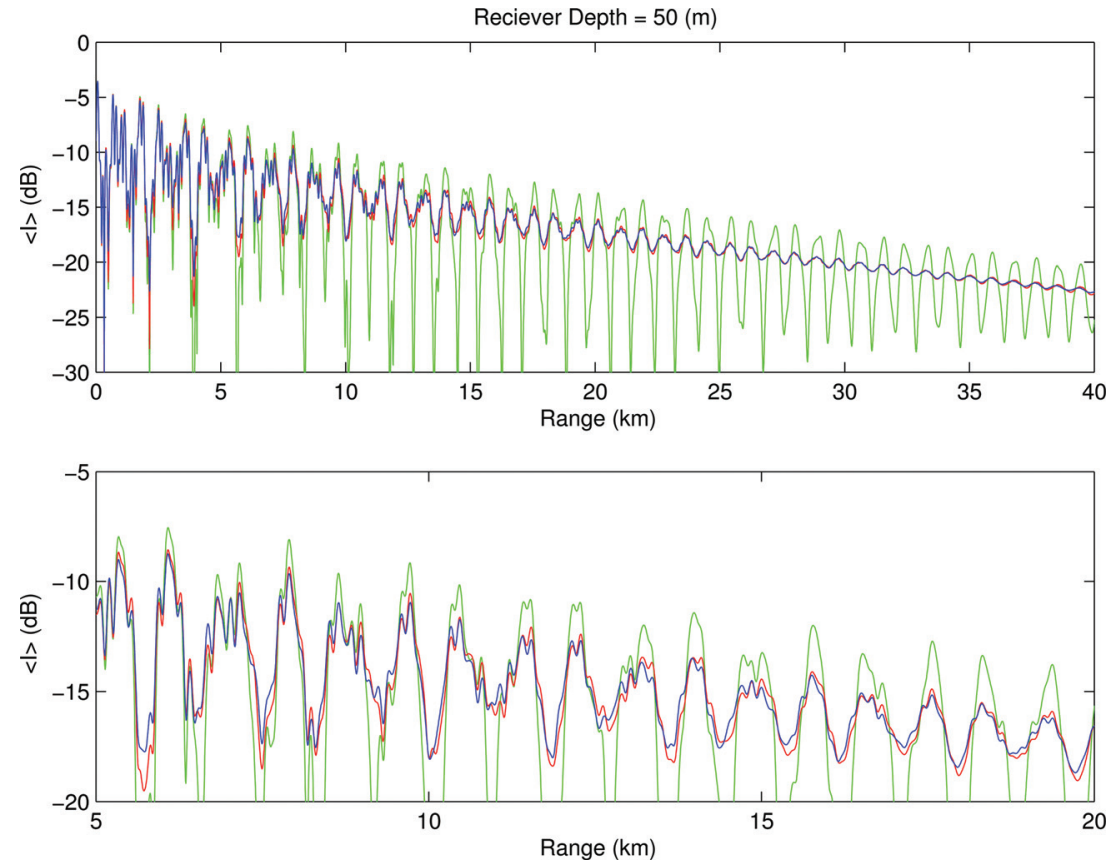

FIG. 3. Mean intensity for $200 \mathrm{~Hz}$ propagation from the Monte Carlo simulation (red), the model results from Eq. (6) (blue), and the unperturbed intensity ( $\mu=0$; green). The lower panels show an expanded view of the range $5-20 \mathrm{~km}$. In each calculation Eq. (4) is utilized, but the cylindrical spreading factor is ignored. The receiver depth is $50 \mathrm{~m}$.

eigenvalues $\lambda_{n}$ are computed; the inverse of the smallest eigenvalue is what Dozier and Tappert term the equipartition range (Dozier and Tappert, 1978a,b). As such it is useful to define an eigenvalue interaction range $R_{n}=\lambda_{n}^{-1}$. Figure 7 plots the mean and minimum of $R_{n}$ for frequencies of 200 , $400,600,800$, and $1000 \mathrm{~Hz}$, where the number of propagating modes is $6,12,18,24$, and 30 . Here it is seen (as has been previously demonstrated) that at the 200 and $400 \mathrm{~Hz}$ frequencies in our example calculations, the propagation is strongly adiabatic with the smallest eigenvalue interaction range being 638 and $116 \mathrm{~km}$, respectively.

For the parameters used in this simulation, Fig. 7 suggests that significant coupling effects would only be observed in the kilohertz frequency range. Again, this
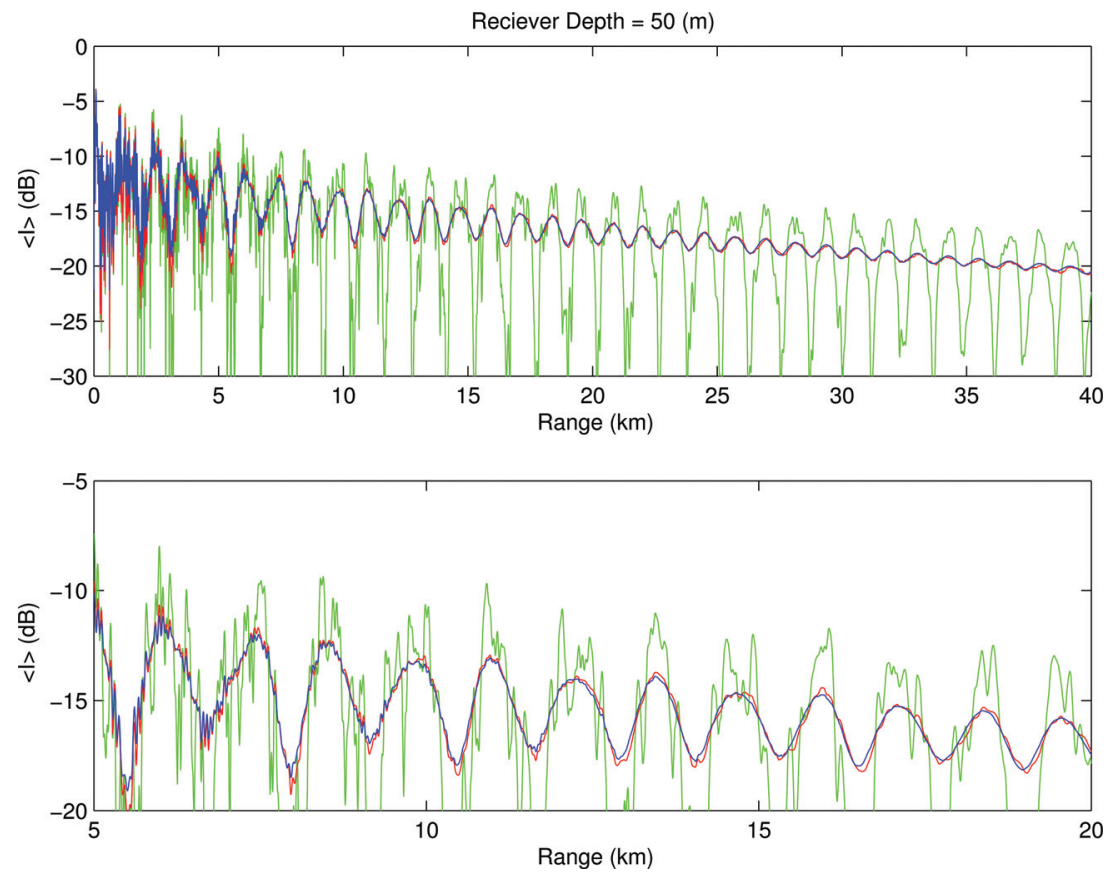

FIG. 4. Mean intensity for $400 \mathrm{~Hz}$ propagation from the Monte Carlo simulation (red), the model results from Eq. (6) (blue), and the unperturbed intensity ( $\mu=0$; green). The lower panels show an expanded view of the range $5-20 \mathrm{~km}$. In each calculation Eq. (4) is utilized, but the cylindrical spreading factor is ignored. The receiver depth is $50 \mathrm{~m}$. 

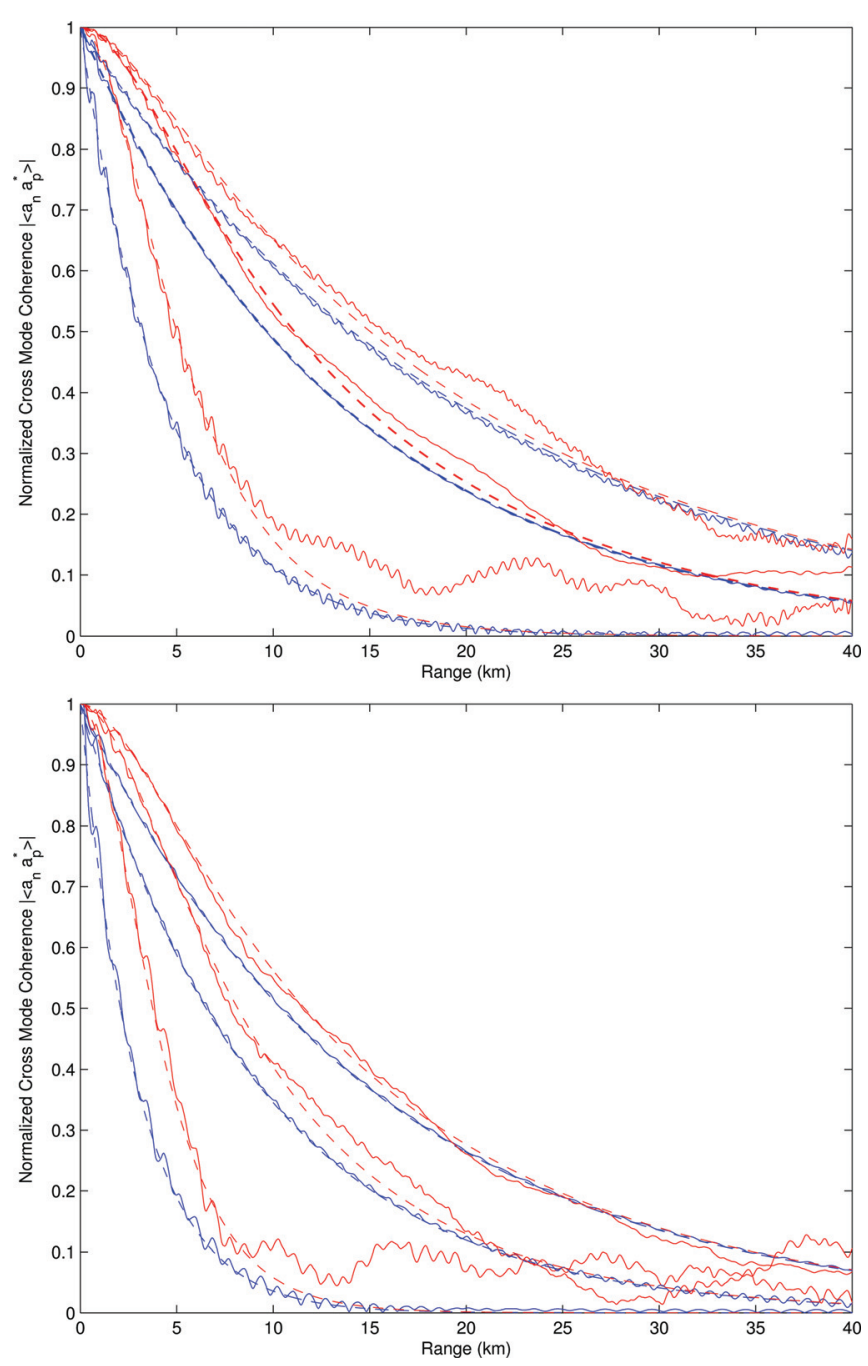

FIG. 5. Range evolution of normalized cross-mode coherences for mode pairs $(1,2),(1,3)$, and $(2,3)$ at 200 (upper panel) and $400 \mathrm{~Hz}$ (lower panel). Monte Carlo numerical simulation results are shown in red, transport equation [Eq. (6)] are shown in blue. The adiabatic results [Eq. (14)] are shown with a blue dash, and a modified adiabatic result, [Eq. (18)], taking account of edge effects is shown with a red dash.

prediction clearly depends on the shallow water environment considered, namely the background profiles, the strength of the perturbations, and the internal-wave spectrum.

\section{E. Higher moments}

The strong adiabaticity of the propagation can be exploited to understand and predict higher moments of the field. The mean square intensity is of fundamental interest because it can be used to provide an "error bar" on the mean intensity. This quantity can be written as

$$
\left\langle I^{2}\right\rangle=\sum_{n} \sum_{p} \sum_{m} \sum_{q} \frac{\left\langle a_{n} a_{p}^{*} a_{m} a_{q}^{*}\right\rangle}{r^{2}} \frac{\phi_{n}(z) \phi_{p}(z) \phi_{m}(z) \phi_{q}(z)}{\sqrt{k_{n} k_{p} k_{m} k_{q}}},
$$

where it is seen that the fourth moment of the mode amplitudes is required. In the adiabatic approximation the mode amplitudes are of the form $a_{n}(r)=a_{n}(0) e^{i\left(l_{n} r+\delta \theta_{n}(r)\right)}$, where $\delta \theta_{n}(r)$ is the adiabatically induced phase fluctuation. If the
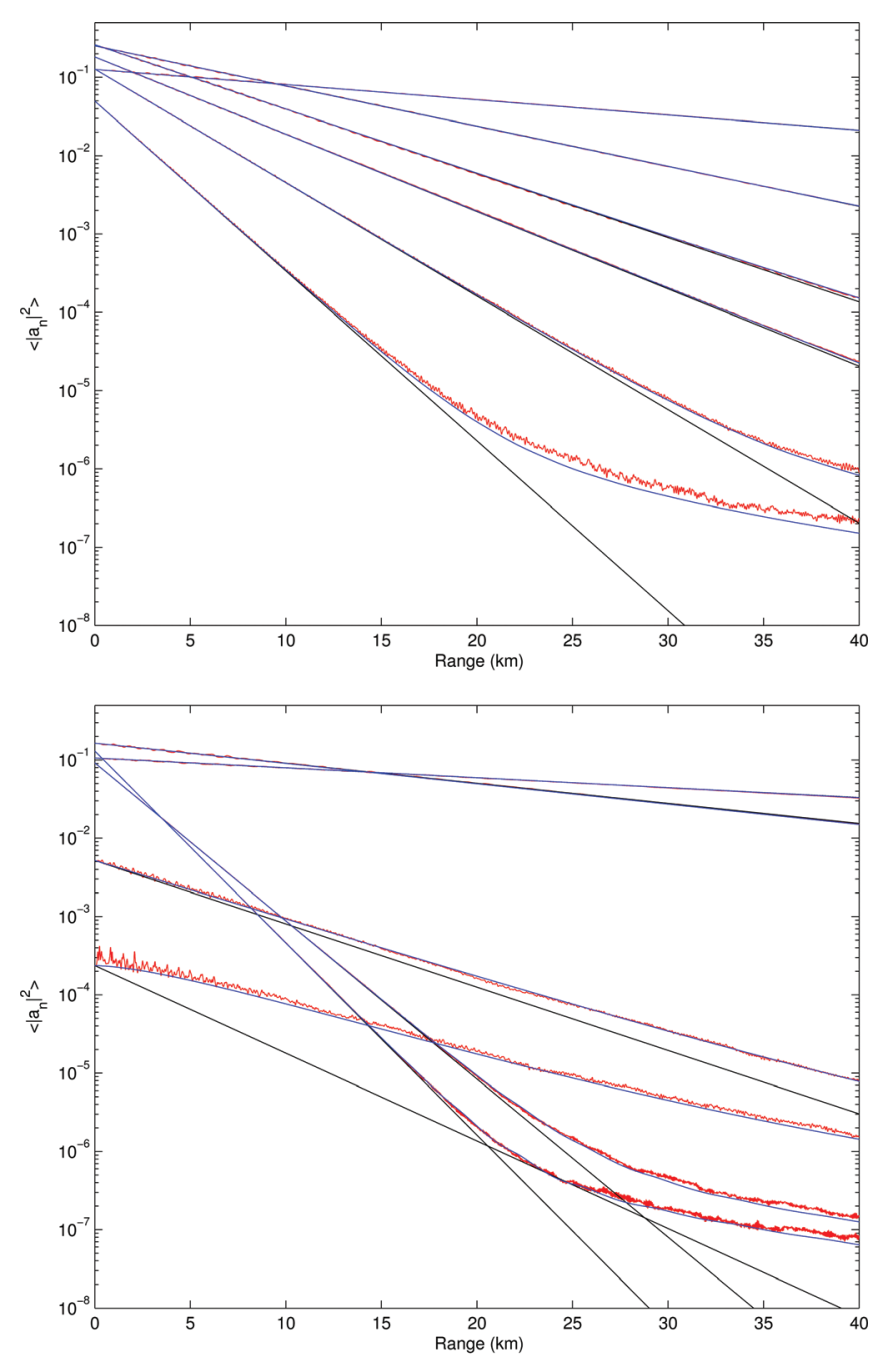

FIG. 6. Range evolution of mean mode energy for 200 (upper panel) and $400 \mathrm{~Hz}$ (lower panel). At $200 \mathrm{~Hz}$, modes 1-6 are displayed, whereas for $400 \mathrm{~Hz}$ modes $1,2,7,8,11$, and 12 are presented. Monte Carlo numerical simulation results are shown in red, transport equations [Eq. (13)] are shown in blue, and adiabatic results [Eq. (14)] are shown in black.

adiabatic phases can be assumed to be Gaussian, as has been done in this paper, a simple calculation yields

$$
\begin{aligned}
\left\langle a_{n} a_{p}^{*} a_{m} a_{q}^{*}\right\rangle= & \hat{a}_{n} \hat{a}_{p}^{*} \hat{a}_{m} \hat{a}_{q}^{*} \exp \left[-\left(I_{n n, n n}+I_{p p, p p}+I_{m m, m m}\right.\right. \\
& +I_{q q, q q}+2\left[I_{n n, m m}+I_{p p, q q}-I_{n n, p p}\right. \\
& \left.\left.\left.-I_{n n, q q}-I_{m m, p p}-I_{m m, q q}\right]\right) r\right]
\end{aligned}
$$

Where $\hat{a}_{n}=a_{n}(0) e^{i l_{n} r}$. Using these results, a comparison between theory and Monte Carlo simulation is carried out using the scintillation index $\mathrm{SI}=\left\langle I^{2}\right\rangle /\langle I\rangle^{2}-1$. Figure 8 shows this comparison for both 200 and $400 \mathrm{~Hz}$, for a receiver depth of $50 \mathrm{~m}$. The adiabatic theory is seen to fit the Monte Carlo data exceptionally well for both frequencies, where it should be noted at short ranges, discrepancies are due to uncorrected edge effects in the adiabatic calculation. The oscillating nature of the scintillation curves is due to the fact that SI is generally larger in the low mean intensity regions and correspondingly SI is smaller in the high mean 
intensity regions. Also of interest is that at long range the scintillation is actually decreasing. This occurs because of mode stripping by attenuation, such that at long range there are fewer high energy modes to interfere and thus cause intensity fluctuations.

\section{IMPLICATION FOR INTERACTION WITH NONLINEAR WAVES}

An important implication of the present work is that for acoustic frequencies on the order of hundreds of hertz, the shallow-water linear internal-wave field adiabatically randomizes the modal phases progressively in range. The decorrelation range can be accurately estimated using Eq. (16), and although some mode pairs have surprisingly large values of $R_{n, p}$, typical values for the low-energy carrying modes are 15 and $30 \mathrm{~km}$ for 400 and $200 \mathrm{~Hz}$, respectively (these values are qualitatively consistent with Figs. 3 and 4). ${ }^{3}$ It has been shown through numerical simulation (Preisig and Duda, 1997; Duda and Preisig, 1999) and through theoretical arguments (Colosi, 2008) that nonlinear internal solitary wave-induced coupling between modes $n$ and $p$ depends critically on the relative phases of the modes at the location of the nonlinear wave. In particular, the modulation factor estimated by single scattering theory (Colosi, 2008) is $\sin \left(k_{n p} r_{0}\right)$ where $r_{0}$ is the location of the wave; that is to say if the relative phase of the modes is an integer multiple of $\pi$ there is no coupling, and if the relative phase is an odd multiple of $\pi / 2$ the transfer of energy between modes is maximum and can be either positive or negative. This being the case if the modal phases are sufficiently variable when they interact with the nonlinear internal wave, the energy is equally likely to couple in any way between the modes with the first-order effect that there is no net coupling on average. The implication then is that the mean intensity will be insensitive to nonlinear internal waves. A further consequence of the phase randomizing effect of the linear internal waves is that after the acoustic field has interacted with a nonlinear wave, the progressive randomization in range will slowly diminish the memory of the nonlinear waves.

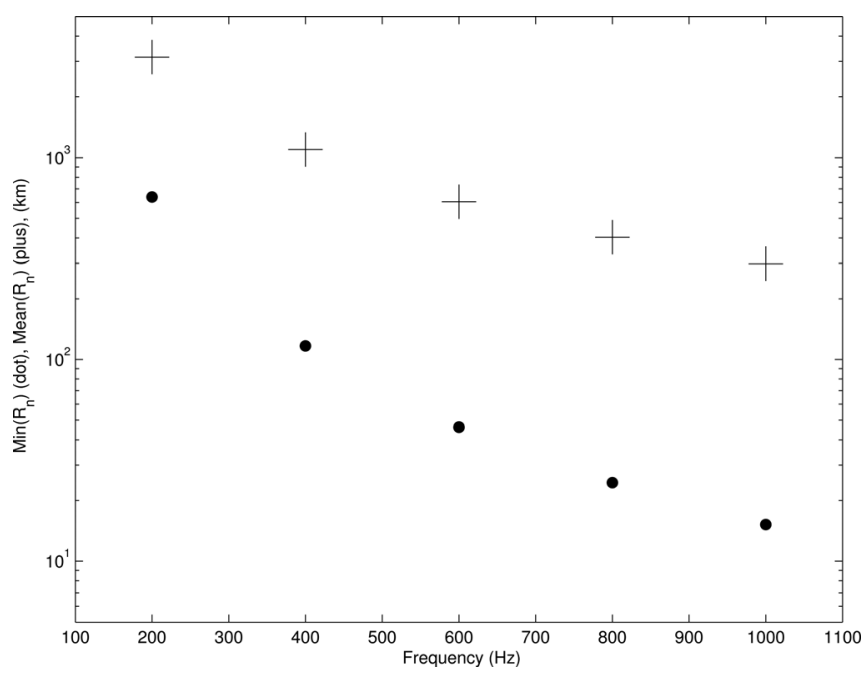

FIG. 7. Mean (plus) and minimum (dot) eigenvalue range $R_{n}$ as a function of acoustic frequency. The minimum of $R_{n}$ scales like frequency to the minus 2 , whereas the mean $R_{n}$ scales like frequency to the minus 1.5 .
Thus, to demonstrate the aforementioned effects, the same Monte Carlo simulations from Sec. III were carried out, but with a sound-speed model of the form $c(r, z)=\bar{c}(z)+\delta c(r, z)+\delta c_{\mathrm{ISW}}(r, z)$, where $\delta c_{\mathrm{ISW}}(r, z)$ is a sound-speed perturbation from a three wave, nonlinear internal-wave packet of the form (Apel et al., 2007)

$$
\delta c_{\mathrm{ISW}}(r, z)=\sum_{n=1}^{3} \zeta_{n} \sin (\pi z / D) \operatorname{sech}^{2}\left(\left(r-r_{n}\right) / \Delta\right) \frac{d \bar{c}}{d z} .
$$

Here the wave amplitudes are $\zeta_{n}=(8,7,6) \mathrm{m}$, the wave widths are $\Delta=100 \mathrm{~m}$, and the wave positions are $r_{n}=(20$, $20.5,21)$ and $(1.0,1.5,2.0) \mathrm{km}$. An example of the combined sound-speed perturbations from the random internal waves and the nonlinear internal waves are shown in Fig. 9. The two different locations of the three-wave packet (i.e., 1.0 and $20.0 \mathrm{~km}$ range) were chosen to cover the cases where the modes were weakly and strongly phase randomized. Note from Fig. 9 that the nonlinear internal-wave packet represents an extremely strong sound-speed perturbation.

\section{A. Mean intensity}

Figure 10 shows the mean intensity patterns for 200 and $400 \mathrm{~Hz}$ propagation through random internal-wave fields with and without a nonlinear internal-wave packet at $1 \mathrm{~km}$ range. Here the modes incident upon the nonlinear waves will be quite coherent, and locally the effect of the wave will be more deterministic. The wave packet is seen to initially shift the interference pattern, but as the range progresses out, the mean intensity is more dramatically shaped by the decorrelation of the modes by the random internal waves. The mean intensity has lost memory of the nonlinear wave. For the $200 \mathrm{~Hz}$ case the mean intensity at longer ranges with the wave packet is seen to be somewhat larger than the case without the wave packet. Apparently the packet has coupled more energy into the lower, less lossy modes. On the other hand, for the $400 \mathrm{~Hz}$ case, the opposite is seen, that is to say the packet has coupled energy into higher mode lossy modes. Such effects of mean attenuation and amplification are consistent with prior model studies (Duda and Preisig, 1999; Duda, 2006).

The situation of a nonlinear internal-wave packet located at the $20 \mathrm{~km}$ range is shown in Fig. 11. In this case the modes incident upon the nonlinear wave have been strongly phase randomized, and the modes are expected to couple with equal likelihood into and out of one another with a net average of zero. This is indeed the case, as the displayed mean intensity in Fig. 10 is quite insensitive to the nonlinear wave packet. Given this situation one may rightly ask the following: over what time span is this mean intensity estimate relevant, given the rapid variations known to exist in the shallow water? To first order one could answer that the time scale is simply the coherence time of the internal waves (on the order of $1 \mathrm{~h}$ ), but a more precise answer would involve examination of the modal coherence times. 

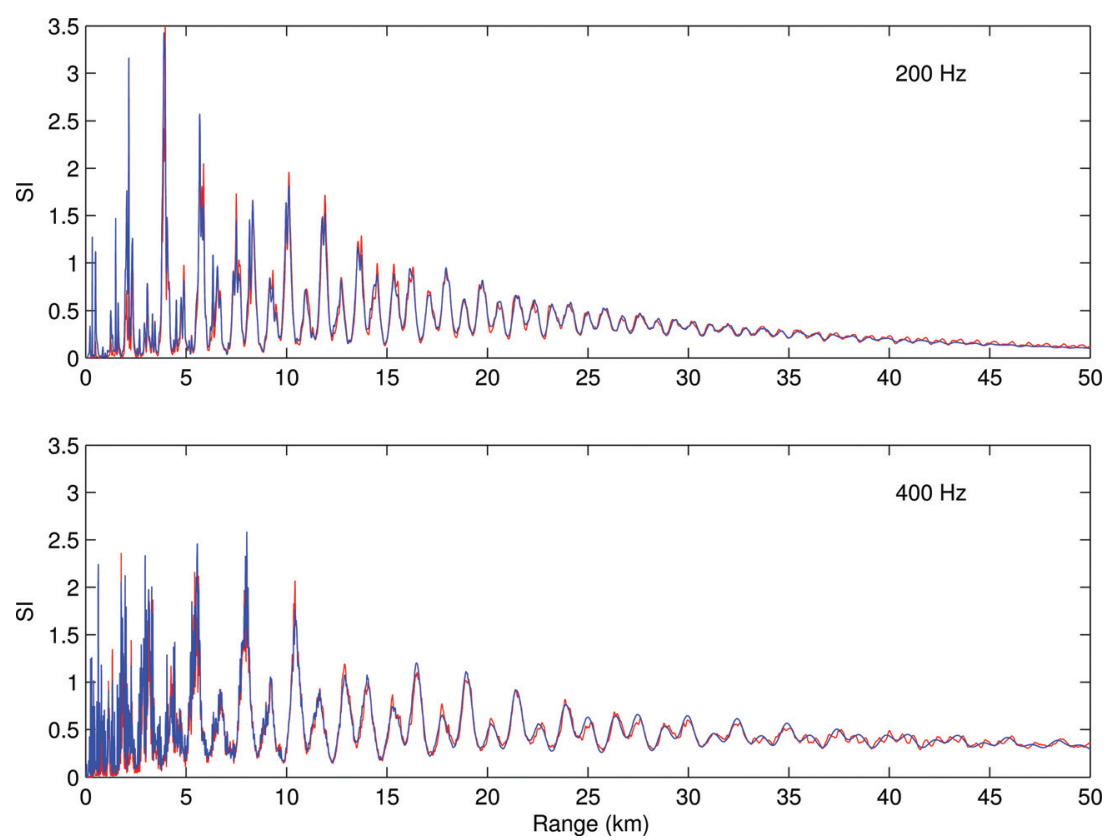

FIG. 8. Range evolution of scintillation index from Monte Carlo simulation (red), and adiabatic theory (blue). The upper/lower panels show 200 and $400 \mathrm{~Hz}$ cases, respectively. The adiabatic theory does not have edge effect corrections.

\section{B. Scintillation index}

The previous results suggest that mean intensity is insensitive to nonlinear internal waves, but is this true of the higher moments, in particular, the scintillation index? Figure 12 shows the comparison of Monte Carlo simulations of scintillation index with and without the three wave nonlinear internalwave packets for the two acoustic frequencies.

For the case of the nonlinear wave near the source (upper panels) there are some small differences in SI due to the shifting of the interference pattern, but the curves are remarkably similar in overall shape and magnitude. Interestingly for the $200 \mathrm{~Hz}$ case the long-range SI values are actually less than the case without the nonlinear waves. This is due to the fact that the nonlinear waves have coupled the more unstable higher mode energy into lower modes as was demonstrated in the mean intensity (see Fig. 10). A similar effect is seen in the $400 \mathrm{~Hz}$ SI curves in which the SI values with the nonlinear waves are slightly higher at long range.
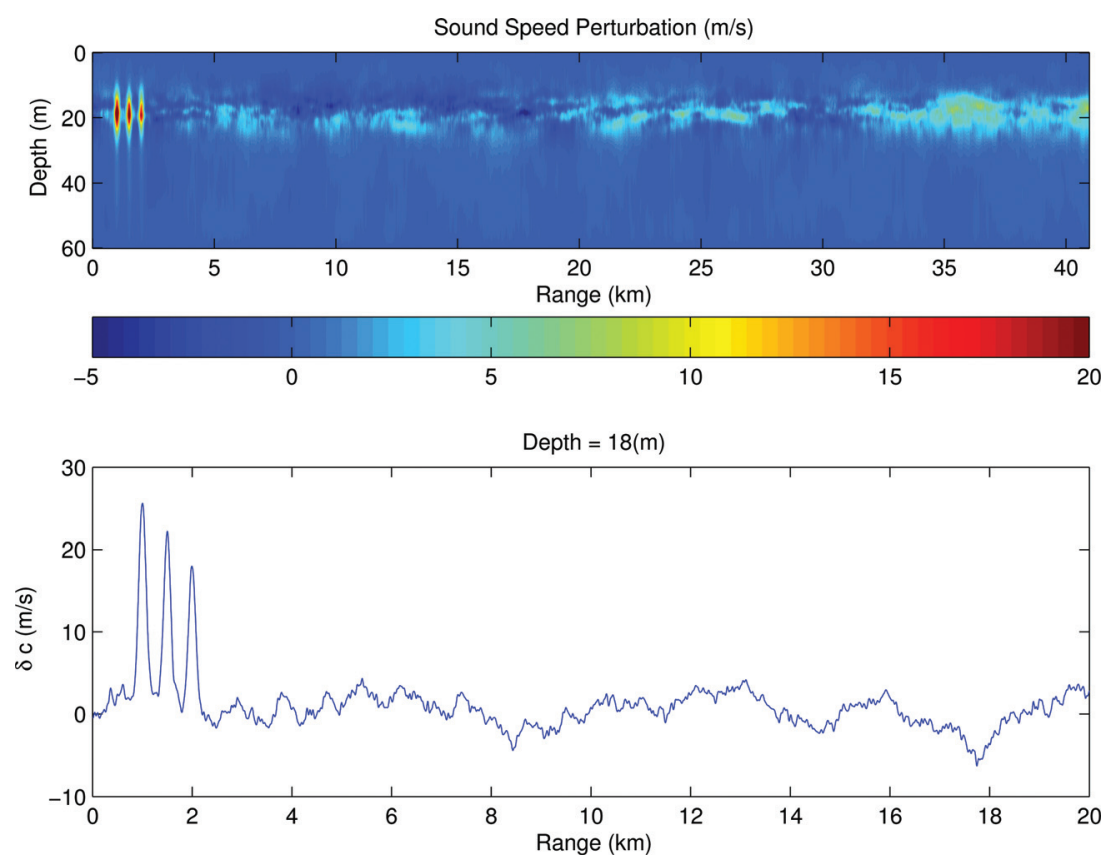

FIG. 9. A realization of sound-speed perturbations from linear and nonlinear internal waves as a function of range and depth (upper panel). The lower panel shows a horizontal slice through this structure at the depth of maximum sound-speed perturbation. 
When the nonlinear internal-wave packet is placed at $20 \mathrm{~km}$ range (lower panels of Fig. 12) it is seen that there is very little impact of the nonlinear wave on the scintillation index. This effect can be understood physically in terms of the loss of coherence of the scattering due to the random waves, that is to say the phase randomization of the modes prevents strong, coherent interference of the modes that would give rise to larger fluctuations. Further study will be required to better understand this interesting effect.

\section{SUMMARY AND CONCLUSIONS}

Second and fourth moments of mode amplitudes and observables of mean and mean square intensity were studied using transport theory, adiabatic theory, and Monte Carlo simulation for a shallow-water environment typical of the summer Mid-Atlantic Bight. For frequencies of 200 and $400 \mathrm{~Hz}$ the propagation is seen to be strongly adiabatic with a rapid decay of cross-mode coherence, whose range scale is of the order of tens of kilometers. Accurate theoretical estimates of cross-mode coherence are obtained only after
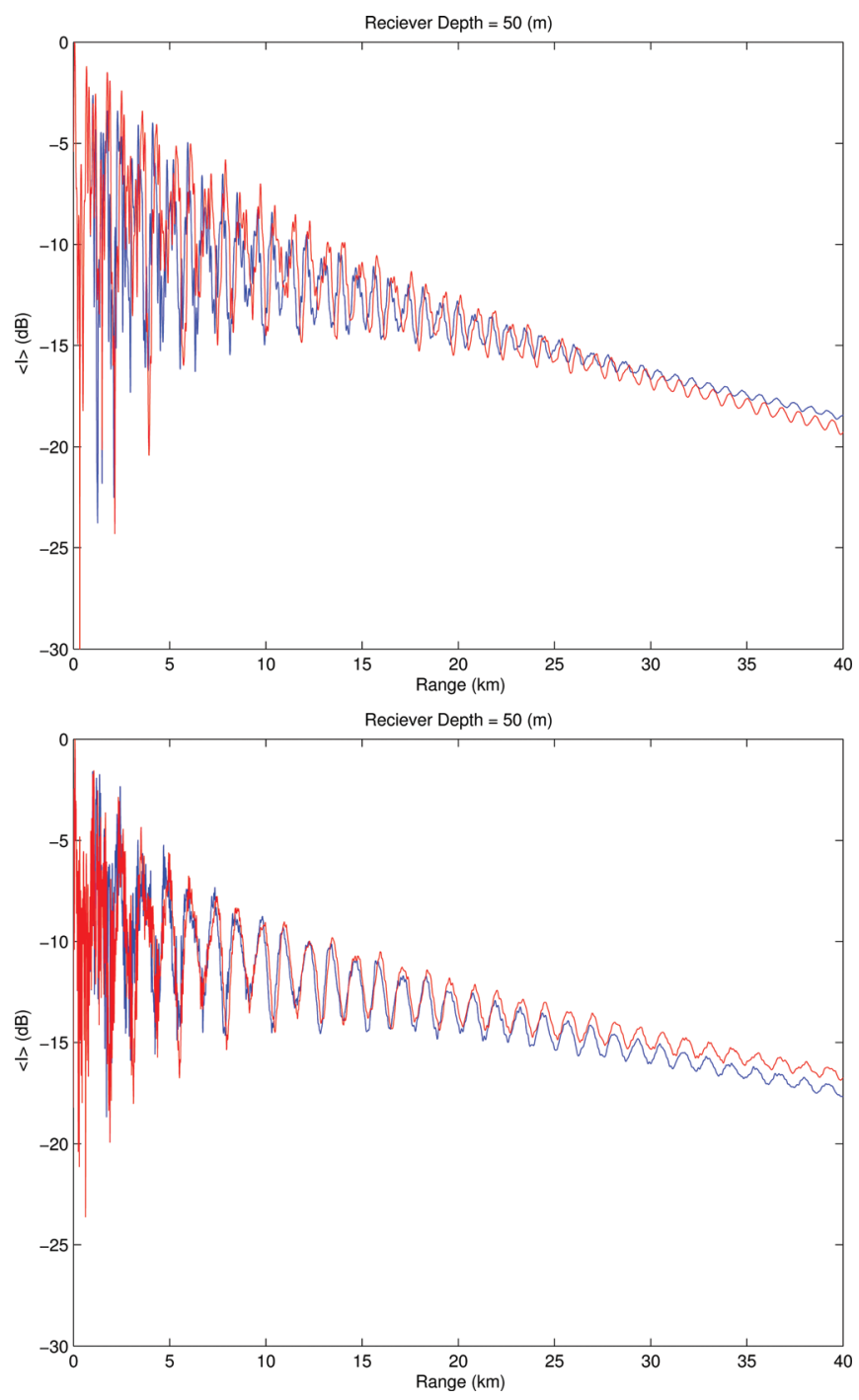

FIG. 10. Mean intensity computed from Monte Carlo simulation with (blue) and without (red) a packet of nonlinear internal waves located at $1 \mathrm{~km}$ range. The upper/lower panels are for $200 / 400 \mathrm{~Hz}$. The receiver depth is $50 \mathrm{~m}$. accounting for edge effects, when the range is comparable to the internal-wave horizontal correlation length. Small coupling effects at these frequencies are correctly modeled using the transport theory, but significant coupling is not expected until kilohertz frequencies.

Mean intensity from transport theory and Monte Carlo simulations are seen to agree within a fraction of a decibel, except at ranges shorter than the horizontal correlation length of the internal waves. Again edge effect corrections to the theory rectify these problems at short range. Because of strong adiabatic cross-mode decorrelation, the modal interference pattern in the mean intensity is significantly smoothed out relative to the unperturbed intensity, pointing to the importance of random linear internal waves to shallow-water acoustic prediction. Further, predictions of mode amplitude fourth moments using adiabatic theory provide predictions of the scintillation index that agree quite well with the Monte Carlo simulations. Therefore, the models described here would seem to provide a very strong low frequency shallow-water model for mean transmission loss and its error bar. Of course there is the issue of the nonlinear
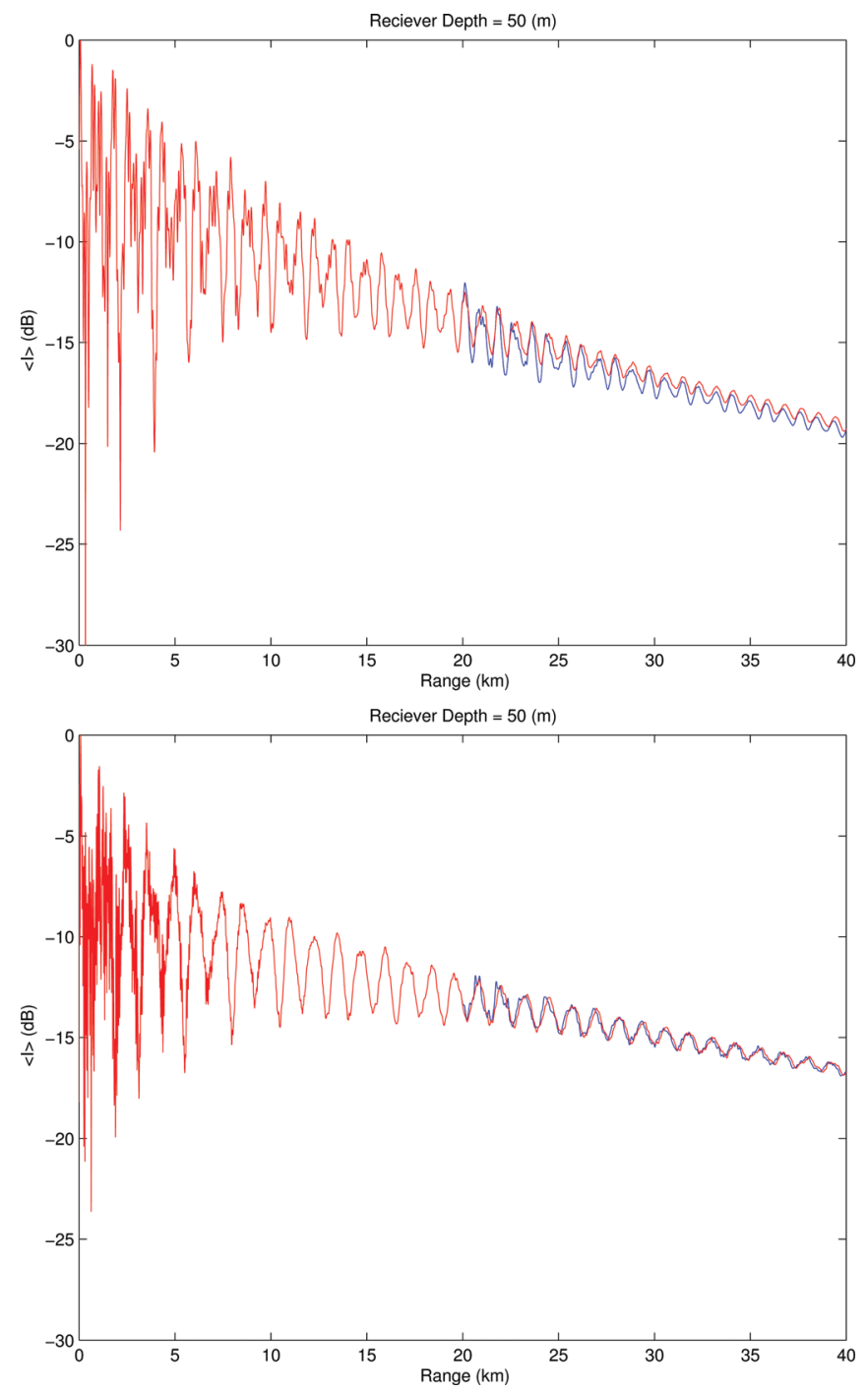

FIG. 11. Mean intensity computed from Monte Carlo simulation with (blue) and without (red) a packet of nonlinear internal waves located at the $20 \mathrm{~km}$ range. The upper/lower panels are for $200 / 400 \mathrm{~Hz}$. The receiver depth is $50 \mathrm{~m}$. 

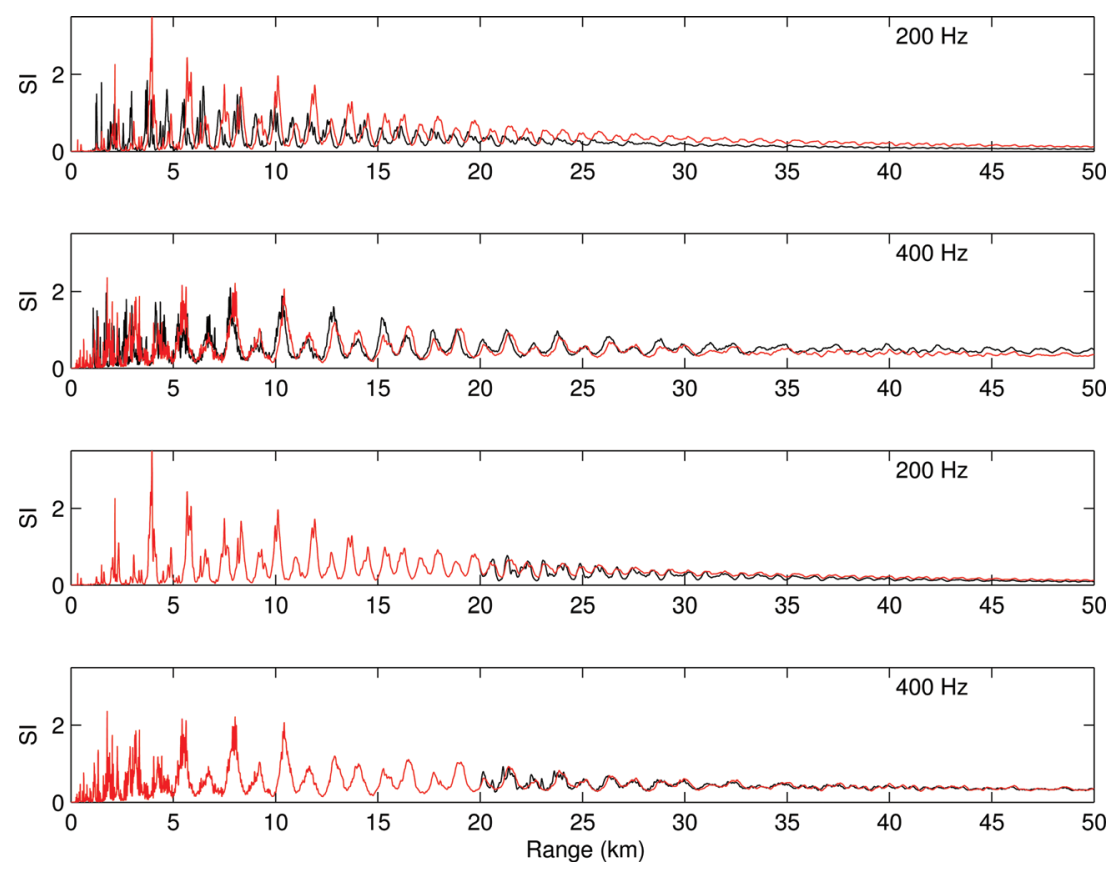

FIG. 12. Range evolution of scintillation index from Monte Carlo simulation with/without nonlinear internal-wave packets (black/red). The upper two panels show the case for the nonlinear wave packet located at the $1 \mathrm{~km}$ range, and the lower two panels are for the case with the waves at the $20 \mathrm{~km}$ range.

internal waves. However, it is shown that strong cross-mode decorrelation and progressive phase randomization dictate that the mean and mean square intensities will be quite insensitive to strong nonlinear internal-wave packet soundspeed perturbations. The conclusions of the Monte Carlo simulation here are indisputable, but further theoretical study will be required to understand this important result in more detail.

It must be pointed out, however, that there are several uncertainties associated with the present results with regards to predicting fluctuations in the real ocean. The foremost issue is that very little information is available concerning the actual shallow-water internal-wave spectrum and its geographic variability. Studies from moored instrumentation on the continental shelf of the Eastern United States (Levine, 2002; Yang and Yoo, 2002; Colosi, 2012) suggest a GMtype spectrum like the one used here is a reasonable model, but there is little or no information on spectral horizontal isotropy, horizontal homogeneity, frequency-mode number factorization, and spectral parameters. In addition, internal waves are not the only source of small-scale sound-speed structure in the shallow water. Spicy thermohaline structure consisting of compensating temperature and salinity anomalies along an isopycnal have been shown to be the dominant source of stochastic sound-speed fluctuation in the summer Mid-Atlantic Bight, and even less is known about the horizontal structure of these features (Colosi et al., 2012).

It must also be kept in mind that the results presented here are expected to have a strong sensitivity to the background profiles of sound speed, density, and attenuation. Within the GM model, one important effect is the stratification influence on the horizontal correlation of the internal waves; a doubling of $N_{0} B$ results in a doubling of the internal-wave correlation length, and thereby a halving of the mode decorrelation range $R_{n, p}$. Other effects are the variation of the important beat wavenumbers $k_{m n}$, the relative mode stripping from attenuation, and the source depth (and thus initial mode energy distribution).

\section{ACKNOWLEDGMENTS}

This work was supported by the Office of Naval Research and the Naval Undersea Warfare Center's UnderSea Warfare chair at the Naval Postgraduate School.

${ }^{1}$ The GM spectrum is a deep water model, but has been used extensively in many shallow water studies for lack of a better model. See, e.g., Rouseff and Tang (2006).

${ }^{2}$ The horizontal correlation length for mode $j$ is approximately $1 / k_{j}$, and thus the total correlation length is $\sum_{j=1}^{J_{m}} H(j) / k_{j}$. For the model of this paper a value of $3.2 \mathrm{~km}$ is obtained, and this value is influenced by the environmental parameter $N_{0} B$.

${ }^{3}$ The occurrence of some large values of $R_{n, p}$ also indicates that the smoothing out of the mean intensity patterns may be a strong function of source depth.

Apel, J., Ostrovsky, L.A., Stepanyants, Y. A., and Lynch, J. (2007). "Internal solitons in the ocean and their effect on underwater sound," J. Acoust. Soc. Am. 121, 695-722.

Beran, M.J., and Frankenthal, S. (1992). "Volume scattering in a shallow channel," J. Acoust. Soc. Am. 91, 3203-3211.

Chen, T., Ratilal, P. and Makris, N. C. (2005). "Mean and variance of the forward field propagated through three-dimensional random internal waves in a continental shelf waveguide," J. Acoust. Soc. Am. 118, 3560-3574.

Colosi, J. A. (2008). "Acoustic mode coupling induced by shallow water nonlinear internal waves: Sensitivity to environmental conditions and space-time scales of internal waves," J. Acoust. Soc. Am. 124, 1452-1464.

Colosi, J. A. and Brown, M. G. (1998). "Efficient numerical simulation of stochastic internal wave induced sound speed perturbation fields," J. Acoust. Soc. Am. 103, 2232-2235.

Colosi, J. A., Duda, T. F., Lin, Y. T., Lynch, J., Newhall, A., and Cornuelle, B. D. (2012). "Observations of sound-speed fluctuations on the New 
Jersey continental shelf in the summer of 2006," J. Acoust. Soc. Am. 131, 1733-1748.

Colosi, J. A., and Morozov, A. (2009). "Statistics of normal mode amplitudes in an ocean with random sound speed perturbations: Cross mode coherence and mean intensity," J. Acoust. Soc. Am. 126, 1026-1035.

Creamer, D.B. (1996). "Scintillating shallow water waveguides," J. Acoust. Soc. Am. 99, 2825-2838.

Dozier, L. B. (1983). "A coupled mode model for spatial coherence of bottom-interacting energy," Proceedings of the Stochastic Modeling Workshop, edited by C. W. Spofford and J. M. Haynes (ARL-University of Texas, Austin, TX).

Dozier, L. B., and Tappert, F. D. (1978a). "Statistics of normal-mode amplitudes in a random ocean. I. Theory," J. Acoust. Soc. Am. 63, 353-365.

Dozier, L. B., and Tappert, F. D. (1978b). "Statistics of normal-mode amplitudes in a random ocean. II. Computations," J. Acoust. Soc. Am. 64, 353-365.

Duda, T., and Preisig, J. (1999). "A modeling study of acoustic propagation through moving shallow water solitary wave packets," IEEE, J. Ocean. Eng. 24, 16-32.

Duda, T.F. (2006). "Temporal and cross range coherence of sound traveling through shallow water nonlinear internal wave packets," J. Acoust. Soc. Am. 119, 3717-3725.

Fredricks, A., Colosi, J. A., Lynch, J. F., Gawarkeiwicz, G., Chiu, C. S., and Abbot, P. (2005). "Analysis of multipath scintillations observed during the summer 1996 New England shelfbreak PRIMER study,” J. Acoust. Soc. Am. 117, 1038-1057.

Headrick, R., Lynch, J., Kemp, J., Newhall, A., von der Heydt, K., Apel, J., Badiey, M., Chiu, C. S., Finnette, S., Orr, M., Pasewark, B., Turgut, A., Wolf, S., and Tielguerger, D. (2000). "Modeling mode arrivals in the 1995 SWARM internal wave scattering experiment," J. Acoust. Soc. Am. 107, 221-236.

Jensen, F. B., Kuperman, W. A., Porter, M. B., and Schmidt, H. (1993). Computational Ocean Acoustics (Springer, New York), Chap. 5.
Levine, M. D. (2002). "A modification of the Garrett-Munk internal wave spectrum," J. Phys. Ocean. 32, 3166-3181.

Lynch, J., and Tang, D. J. (2008). "Overview of shallow water 2006 JASA EL Special Issue Papers,” J. Acoust. Soc. Am. 124, EL63-EL65.

Munk, W. (1981). "Internal waves and small scale processes," in The Evolution of Physical Oceanography, edited by B. Warren and C. Wunsch (MIT, Cambridge Press, Cambridge, MA), 264-291.

Preisig, J., and Duda, T. (1997). "Coupled acoustic mode propagation through continental-shelf internal solitary waves," IEEE J. Ocean. Eng. 22, 256-269.

Ratilal, P., and Makris, N. C. (2005). "Mean and covariance of the forward field propagated through a stratified ocean waveguide with three dimensional random inhomogeneities," J. Acoust. Soc. Am. 118, 3532-3559.

Rouseff, D., and Tang, D. (2006). "Internal wave effects on the ambient noise notch in the East China Sea: Model/data comparison," J. Acoust. Soc. Am. 120, 1284-1294.

Sazontov, A. G., Matveyev, A. L., and Vdovicheva, N. K. (2002). "Acoustic coherence in shallow water: Theory and observation," IEEE J. Ocean. Eng. 27, 653-663.

Tielburger, D., Finnette, S., and Wolf, S. (1997). "Acoustic propagation through an internal wave field in a shallow water waveguide," J. Acoust. Soc. Am. 101, 789-808.

Van Kampen, N. G. (1981). Stochastic Processes in Physics and Chemistry (North-Holland, New York), Chap. 16

Voronovich, A. G., and Ostashev, V. E. (2009). "Coherence function of a sound field in an ocean with horizontally isotropic statistics," J. Acoust. Soc. Am. 125, 99-110.

Yang, T. C., and Yoo, K. (2002), "Internal wave spectrum in shallow water: measurement and comparison with the Garrett-Munk model," IEEE J. Ocean. Eng. 24, 333-345.

Zhou, J., Zhang, X., and Rogers, D. (1991). "Resonant interaction of sound with internal solitons in a coastal zone," J. Acoust. Soc. Am. 90, 2042-2054. 